\title{
Implementations of UKF and EKF for Wheel Slip Control Purpose to Locomotive Computer
}

\author{
Petr Pichlík \\ Dept. of Electric Drives and Traction \\ Czech Technical University in Prague, Faculty of Electrical Engineering \\ Technická 2, 16627 Prague, Czech Republic \\ pichlpet@fel.cvut.cz
}

\begin{abstract}
Modern electric locomotives have to transfer high tractive effort between wheels and rails. Therefore, they have to be equipped with slip controllers that enable to achieve required force and keep wheelsets velocity in the acceptable value. The slip controller is a part of the locomotive control algorithm that runs on an axle computer that is typically a digital signal processor with limited computational power. The modern slip controller requires determining an actual value of adhesion that cannot be directly measured during the train runs. Therefore, the adhesion estimation is necessary. Extended Kalman filter or unscented Kalman filter can be used with an advantage because they can cope with some drawbacks of conventional methods. However, they are not typically used because of their high computational requirements. Therefore, the extended Kalman filter and unscented Kalman filter are implemented into a digital signal processor that is typically used in the locomotives as an axle computer to verify the possibility of used slip control methods based on the mentioned estimators in the paper. The verification is made on measured data.
\end{abstract}

Keywords-slip control; extended Kalman filter; unscented Kalman filter; railway.

\section{INTRODUCTION}

Modern electric locomotives are complex mechanical and electrical system, and for their control, significant computing power is required. The required computing power provides a locomotive computer that is designed as a distributed computer [1], [2]. The computer consists of a master computer and computers for electric drives and auxiliary devices. The computers for electric drives are typically digital signal processors (DSP), and every electric drive is controlled by its DSP. If more motors are connected to one inverter, the DSP control the group. However, this configuration is not typical in modern locomotives [3], but it is possible in electric multiple units [4] or older locomotives [5]. The computer connected with the wheelset has to provide control of the electric drive. The computer also calculates a slip controller that is an integral part of the electric drive control for railway vehicles. The computer also provides measurement and communication with the master computer.

The slip controller is the essential part of the electric drive control because it limits the wheel slip velocity to acceptable value if the high tractive force is required. The slip velocity is needed for a force transfer between wheels and rails. Value of the maximum transferable force and slip velocity mainly depends on the conditions of a rail surface that change during the train runs. The dependence of the actual adhesion on the slip is described by a nonlinear characteristic. The slip is a ratio of the slip velocity and train longitudinal velocity. The slip velocity starts to increase when the applied force to the wheels is higher than the maximum force that can be transferred between wheels and rails. In this case, the tractive force has to be decreased. The high slip velocity value is undesirable because increases wear of wheels and rails [6] and can damage some locomotive components. The modern slip controllers try to prevent the high slip velocity creation. The slip controllers can be sorted into controllers that try to find the maximum value of the current characteristic [7] or methods that try to determine the slope of the characteristic. The slope is typically calculated by using a disturbance observer [8]. However, Kalman filter, extended Kalman filter (EKF) or unscented Kalman filter (UKF) can also be used [9], [10].

The estimators can be divided into early estimators, modern estimators and disturbance observers [11]. The typical member of the early estimator is a Luenberger observer. The typical member of the modern observes the Kalman filter, EKF or UKF. All of the estimators can be used for the slip controller purpose, and there were efforts of implementation of all estimators. The most used types of the estimators are disturbance observers. However, the method based on the EKF or UKF provides accurate results but their design complexity is high [11], and their computational complexity is also high especially the UKF. On the other hand, the increasing computational power of modern microcontrollers allows implementing the EKF or UKF into a control algorithm.

The rest of the paper is organized as follows. Firstly, an appropriate model of a locomotive axle that is used by the estimator is described. Next, the algorithm of the EKF and UKF are presented. Then, the implementation of the slip controller implementation into the control algorithm and the estimator implementation into the slip controller is presented. Finally, the implantation performance into a DSP and simulation results are presented on measured data. 


\section{ESTIMATORS IMPLEMENTATION}

\section{A. Mathematical Model}

The mathematical model is the essential part of the estimator. The disturbance observers very often use a simple model that is based on the equation of motion. This model is used because of its low computational power requirements. However, the model cannot provide such accurate results as more complex models. Therefore, a two-mass model is used for the estimator. The two-mass model can provide more precise results and enables to preserve one of the eigenfrequencies that occur in the axle. The first eigenfrequency is the frequency between an electric motor and the wheelset and the second is between wheels on the wheelset. The values of the frequencies depend on the mechanical components parameters. However, its computational requirements are too high. The general schema of a simple one locomotive axle is depicted in Fig. 1, and the schema for the two-mass model is depicted in Fig. 2. The locomotive axle model consists of an electric motor, gearbox, directly driven wheel and indirectly driven wheel. Every mass is represented by its moment of inertia, and the shafts are represented by its stiffness and damping. The two-mass model consists of the electric motor and the wheel mass with the gearbox mass.

The two-mass model state equations are:

$$
\begin{gathered}
\frac{\mathrm{d}}{\mathrm{d} t} \mathrm{x}=\mathrm{Ax}+\mathrm{Bu}+w \\
\mathrm{y}=\mathrm{Cx}+v
\end{gathered}
$$

Where $\mathrm{B}$ is an input matrix, $\mathrm{C}$ is an output matrix, $\mathrm{u}$ is an input vector, $y$ is an output vector, $w$ and $v$ are random noises. The state vector $x$ and state matrix A are defined as follows.

$$
\begin{gathered}
\mathrm{x}=\left[\begin{array}{c}
\varphi_{\mathrm{D}-\mathrm{W}} \\
\omega_{\mathrm{M}} \\
\omega_{\mathrm{W}} \\
i
\end{array}\right] \\
\mathrm{A}=\left[\begin{array}{cccc}
0 & -1 & -1 & 0 \\
\frac{c_{\mathrm{MW}} \cdot J_{\mathrm{M}}}{J_{\mathrm{W}}} & \frac{d_{\mathrm{MW}} \cdot J_{\mathrm{M}}}{J_{\mathrm{W}}} & \frac{-d_{\mathrm{MW}} \cdot J_{\mathrm{M}}}{J_{\mathrm{W}}} & \frac{r_{\mathrm{G}}}{J_{\mathrm{W}}} \\
-0 & 0 & 0 & 0
\end{array}\right](4)
\end{gathered}
$$

Where $\varphi_{\mathrm{D}-\mathrm{W}}$ is a difference angle in the different ends of the shaft, $\omega_{\mathrm{M}}$ is the motor angular velocity, $\omega_{\mathrm{W}}$ is the wheel angular velocity, $i$ is estimated adhesion, $c_{\mathrm{MW}}$ and $d_{\mathrm{MW}}$ is a stiffness and damming of the shaft between the motor and the wheel, $J_{\mathrm{M}}$ and $J_{\mathrm{W}}$ are moments of inertia of the motor and wheel respectively and $r_{\mathrm{G}}$ is a gear ratio of the gearbox.

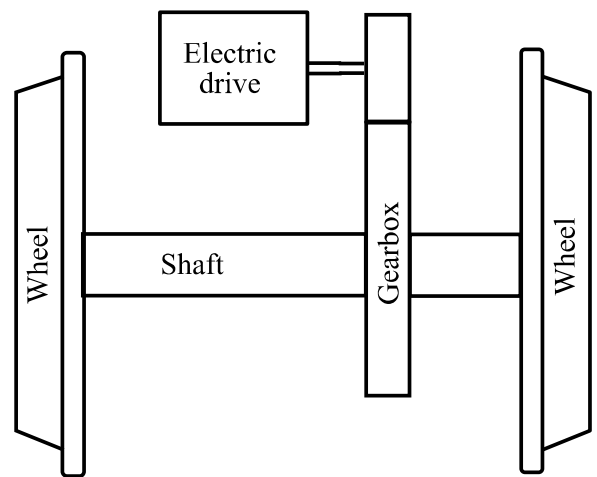

Figure. 1 General schema of one locomotive axle

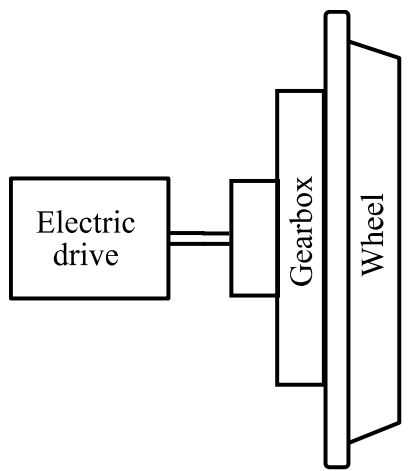

Figure. 2 Two-mass model schema

\section{B. Estimators}

The model of mechanics is linear. However, the interaction in wheel-rail contact is nonlinear due to the adhesion. Typical dependences of an adhesion coefficient on the slip velocity are depicted in Fig. 3. The shape of the characteristic changes with rails surface conditions that complicate the nonlinearity description. The factors that influence the characteristic are, e.g., in [12]. The dependence is nonlinear and can cause failure of some slip controllers. Therefore, the characteristic should be taken into account and using estimators for linear systems may not be appropriate although they are used.

If the nonlinearity is taken into account, the state equations change to form:

$$
\begin{gathered}
\frac{\mathrm{d}}{\mathrm{d} t} \mathrm{x}=f(\mathrm{x})+\mathrm{Bu}+w \\
\mathrm{y}=h(\mathrm{x})+v
\end{gathered}
$$

Where $f$ and $h$ are nonlinear functions.

Equations (5) and (6) do not contain the matrixes $\mathrm{A}$ and $\mathrm{C}$. However, the matrixes have to be known for the EKF calculation. Therefore, the matrixes have to be calculated by calculating Jacobians during the EKF calculation. The known EKF algorithm is presented, e.g. in [13] in detail. The EKF calculates an approximation of the nonlinear function. Therefore, the EKF can diverge due to the linearization [14]. The algorithm of the UKF is more complicated than the algorithm of the EKF, but it does not calculate the approximation. The algorithm is described in [13]. 


\section{Program Structure}

The program structure of a typical electric drive control algorithm is described in Fig. 4. The program runs in the DSP that is intended for one wheelset. The main part is the electric drive control that is mostly a vector control nowadays. However, any other type of control can be used. The DSP also has to provide a measurement of analogue quantities and speed measurement. These measurements are made due to the vector control. The required torque and other command are received from the master computer. The slip controller is a part of the algorithm, but typically it is a separate algorithm that influences only the vector control input. The slip controller typically needs a motor or wheelset velocity and motor torque for its correct function. The slip controller that estimates the characteristic slope consists of the characteristic slope detection part that is created from the EKF or UKF and forms the controller detection part. The second part is a controller that can be any type. The principle of the detection part is output, and its processing by the controller part is described in [15].

The main problem of sophisticated algorithms of the slip controllers is in the available calculation time. The control loop of the whole algorithm can be $400 \mu \mathrm{s}$, and the slip controller can take maximally $100 \mu \mathrm{s}$. This time is enough for simple slip control method, e.g. based on the disturbance observer, but for sophisticated algorithms, the time can be strongly limited, and it requires fast DSP.

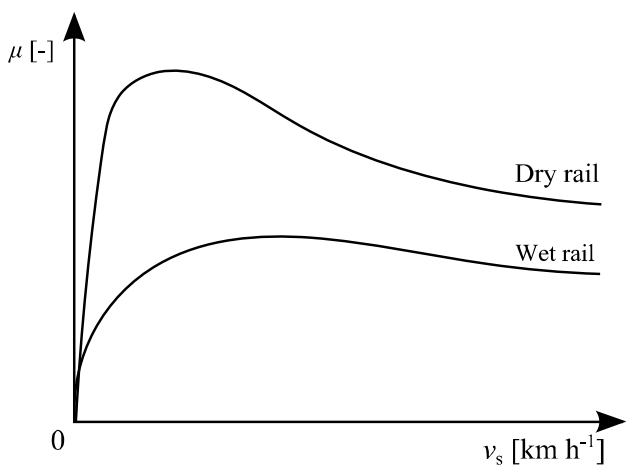

Figure. 3 Example of the dependence of the adhesion coefficient on the slip velocity.

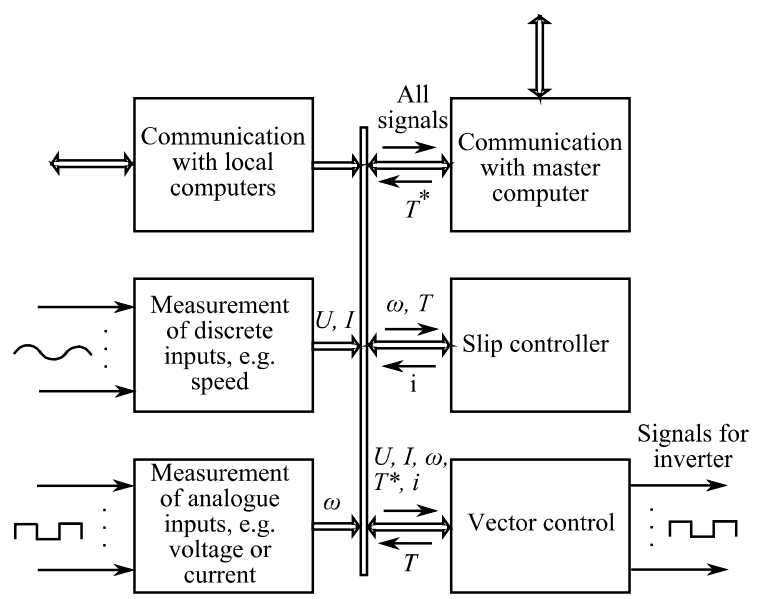

Figure. 4 Block diagram of the program structure

\section{SIMULATION RESULTS AND IMPLEMENTATION}

The reaction of the EKF and UKF to the high slip velocity is depicted in Fig. 5. The measured wheel velocity and tractive force applied to the wheelset is depicted at the top of the figure. The high slip velocity is depicted in the figure. Because the data are measured, the response of the re-adhesion controller is depicted in the figure. The re-adhesion controller decreased the tractive force to eliminate the high value of the slip velocity. The re-adhesion controller is a part of the control structure of the locomotive, but its reaction is improper because the slip velocity value is too high. The calculated output of the EKF and UKF is depicted at the bottom part of the Fig. 5. The values are almost the same as the part where the slip velocity has low value. The slight difference occurs when the high value of the slip velocity occur. However, the difference is not significant.

The comparison of calculation of the estimation part of the UKF by a Matlab and DSP are shown in Fig. 6. There are shown wheelset velocity with high slip velocity value and tractive force as in Fig. 5 and the corresponding estimated relative adhesion force that is calculated by the UKF. There is no significant difference between the calculated signals by the Matlab and DSP.

The required time for the calculation of the EKF and UKF are given in Table 1 . The required times are for the proposed model. The UKF requires almost 2.5 times more calculation time than the EKF. This fact limits the possible usage of the UKF on the DSP with lower computational power. On the other hand, the UKF can provide more stable results than the EKF. The required time was measured after the EKF and UKF implementation to the DSP TMS300F28335 with machine cycle $6.67 \mathrm{~ns}$. The required time is higher than the time calculated by multiplication of the number of operation by the machine cycle because some operations take more time. The separation into a required time with nonlinear function and with the nonlinear function is made because the nonlinear function can be any type.
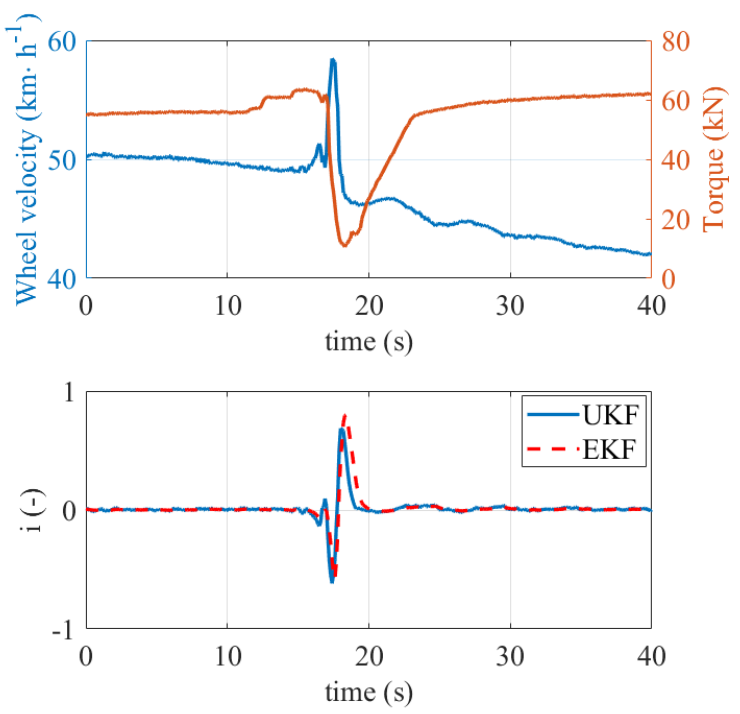

Figure. 5 Comparison of calculation by the EKF and UKF by Matlab 

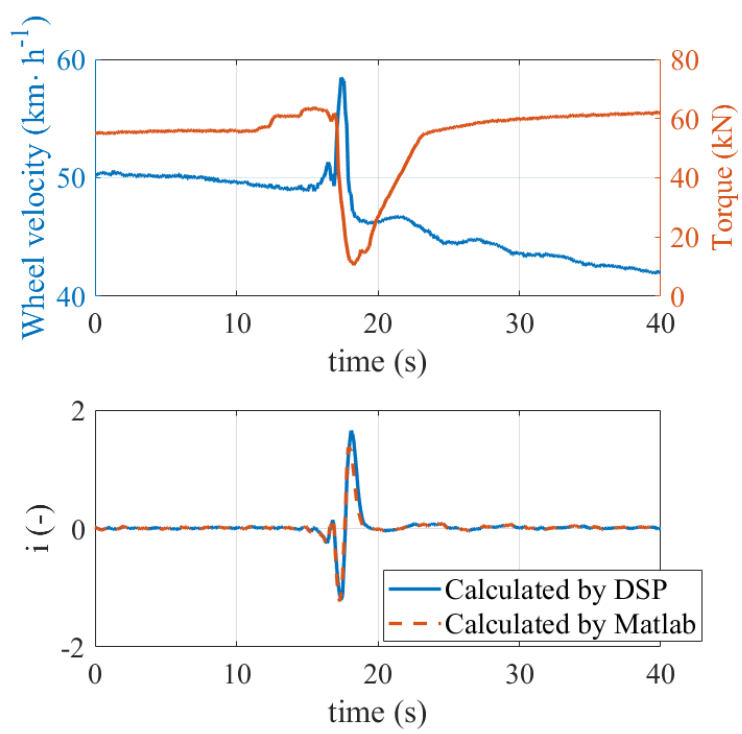

Figure. 6 Comparison of calculation by Matlab and DSP

Table 1 Comparison of the EKF and UKF required calculation time

\begin{tabular}{|c|c|c|c|c|}
\hline \multirow{2}{*}{ Filter } & \multicolumn{2}{|c|}{ Number of operation (-) } & \multicolumn{2}{c|}{ Required time $(\boldsymbol{\mu s})$} \\
\cline { 2 - 5 } & $\begin{array}{c}\text { Without } \\
\text { nonlinear } \\
\text { function }\end{array}$ & $\begin{array}{c}\text { With } \\
\text { nonlinear } \\
\text { function }\end{array}$ & $\begin{array}{c}\text { Without } \\
\text { nonlinear } \\
\text { function }\end{array}$ & $\begin{array}{c}\text { With } \\
\text { nonlinear } \\
\text { function }\end{array}$ \\
\hline EKF & 3786 & 5095 & 28.5 & 38.3 \\
\hline UKF & 12148 & 13520 & 85.6 & 95.3 \\
\hline
\end{tabular}

\section{CONCLUSIONS}

The paper presents comparison and possibility of using the EKF and UKF as the estimator for the locomotive slip controller. The estimator is required for estimation of the adhesion force that cannot be measured during the train runs. The force can be estimated by the disturbance observers that are used because of their low computational requirements. However, the EKF or UKF are not used due to their high computational complexity. Both, EKF and UKF are compared in the paper, and their computational complexity is demonstrated on an example of the twomass model of one locomotive axle. The high computational complexity of the slip controller is problematic because the slip controller runs in the DSP that have to calculate the electric drive control and related parts. The slip controllers are often considered as a secondary control program. However, they are the important part that enables to transfer maximal force and keep the slip velocity at the acceptable value. The EKF and UKF take $38.3 \mu$ s and $95.3 \mu$ s respectively. The computational complexity of the UKF is significantly higher but the time is below $100 \mu \mathrm{s}$, and the slip controller based on the UKF can be used. The paper also presents the two-mass model that is used as the model for the estimator and program structure in the DSP. The two-mass model consists of the electric drive that is represented by electric motor and wheel with the gearbox. The DSP has to calculate the vector control, slip controller, provide the measurement of analogue and digital signals, generate signals for the inverter and communicate with other computers.

\section{ACKNOWLEDGMENT}

This work was supported by the Technology Agency of the Czech Republic under the grant No. TE02000103, Center for Intelligent Drives and Advanced Machine Control.

\section{REFERENCES}

[1] J. Zdenek, "Traction vehicle distributed control computer system architecture with auto reconfiguration features and extended DMA support," 2008 13th International Power Electronics and Motion Control Conference, Poznan, 2008, pp. 1638-1645.

[2] J. Zdenek, "Distributed control computer backbone communication channel of electric locomotive with effective DMA support," Proceedings of 14th International Power Electronics and Motion Control Conference EPE-PEMC 2010, Ohrid, 2010, pp. T6-27-T6-34.

[3] M. Yamashita and T. Soeda, "A novel slip control method considering axle-weight transfer for electric locomotive," 2010 IEEE Vehicle Power and Propulsion Conference, Lille, 2010, pp. 1-6.

[4] K. Kondo, "Anti-slip control technologies for the railway vehicle traction," 2012 IEEE Vehicle Power and Propulsion Conference, Seoul, 2012, pp. 1306-1311.

[5] P. Pichlík, O. Zoubek and J. Zděnek, "Measuring device for measurement of train dynamic motion during wheel slip," 2014 International Conference on Applied Electronics, Pilsen, 2014, pp. 247-250,

[6] X. Cao et al., "The effect of alumina particle on improving adhesion and wear damage of wheel/rail under wet conditions", Wear, vol. 348-349, pp. 98-115, Feb. 2016.

[7] S. Sadr, D. A. Khaburi and J. Rodríguez, "Predictive Slip Control for Electrical Trains," in IEEE Transactions on Industrial Electronics, vol. 63, no. 6, pp. 3446-3457, June 2016.

[8] W. Lin, Z. Liu, L. Diao, G. Zhang, D. Chen and Z. Li, "Maximum Adhesion Force Control Simulated Model of Electric Locomotive," 2007 IEEE International Conference on Automation and Logistics, Jinan, 2007, pp. 1704-1708.

[9] T. Ishrat, G. Ledwich, M. Vilathgamuwa and P. Borghesani, "Identification scheme of maximum traction force using recursive least square for traction control in electric locomotives," 2017 IEEE 12th International Conference on Power Electronics and Drive Systems (PEDS), Honolulu, HI, 2017, pp. 1,120-1,125.

[10] P. Pichlík and J. Zděnek, "Comparison of a Locomotive Adhesion Force Estimation Methods for a Wheel Slip Control Purpose," 2017 9th International Conference on Electronics, Computers and Artificial Intelligence (ECAI), 2017, pp. 1-4

[11] A. Radke and Zhiqiang Gao, "A survey of state and disturbance observers for practitioners," 2006 American Control Conference, Minneapolis, MN, 2006, pp. 6

[12] C. Bernsteiner, G. Muller, A. Meierhofer, K. Six, D. Kunstner and P. Dietmaier, "Development of white etching layers on rails: simulations and experiments“, Wear, vol. 366-367, 2016, pp. 116-122

[13] M. S. Grewal, A. P. Andrews, Kalman Filtering Theory and Practice Using MATLAB. 3rd edition, Wiley, New York 2008.

[14] E. A. Wan and R. Van Der Merwe, "The unscented Kalman filter for nonlinear estimation," Proceedings of the IEEE 2000 Adaptive Systems for Signal Processing, Communications, and Control Symposium, Lake Louise, Alta., 2000, pp. 153158.

[15] P. Pichlík and J. Zděnek, "Comparison of locomotive adhesion force estimation methods for a wheel slip control purpose," 2017 9th International Conference on Electronics, Computers and Artificial Intelligence (ECAI), Targoviste, 2017, pp. 1-4. 